\title{
An assessment of the newest magnetar-SNR associations
}

\author{
J. E. Horvath * \\ M. P. Allen $\diamond$ \\ * Instituto de Astronomia, Geofísica e Ciências Atmosféricas - Universidade de São Paulo \\ Rua do Matão, 1226, 05508-900, Cidade Universitária, São Paulo SP, Brazil \\ $\diamond C E F E T / S P$, R. Pedro Vicente 625, Canindé, São Paulo SP, Brazil
}

\begin{abstract}
Anomalous X-ray Pulsars and Soft-Gamma Repeaters groups are magnetar candidates featuring low characteristic ages $\left(\tau=\frac{P}{2 P}\right)$. At least some of them they should still be associated with the remnants of the explosive events in which they were born, giving clues to the type of events leading to their birth and the physics behind the apparent high value of the magnetar magnetic fields. To explain the high values of $B$, a self-consistent picture of field growth also suggests that energy injection into the SNR is large and unavoidable, in contrast with the evolution of conventional SNR. This modified dynamics, in turn, has important implications for the proposed associations. We show that this scenario yields low ages for the new candidates CXOU J171405.7-381031/CTB 37B and XMMU J173203.3-344518/G353.6-0.7, and predicted values agree with recently found $\dot{P}$, giving support to the overall picture.
\end{abstract}

Keywords: SNR - Magnetars

\section{Energy injection in magnetar-driven SNRs}

Several studies of the expansion of remnants in different ISM were performed over the years, just assuming that the explosion is successful an an energy of $\sim 10^{51} \mathrm{erg}$ 14 is released in a point-like region and unrelated to the compact remnant. Such vision was first challenged by Ostriker and Gunn 18 when they postulated that the rotation energy of a central pulsar may drive the supernova. Later it became clear that this phenomenon is real but too slow to power explosions, although the so-called magnetodynamical mechanisms 6 still retain many of these features.

The identification of superstrong magnetic fields in magnetars posed yet a further related problem to the explosion scenarios. If the field had to be amplified from an initial seed, then the pre-supernova progenitors must have a suitable distribution of both magnetic field and angular momentum. If the initial rotation is not fast enough, the amplification of the field is quenched 19. This leads to think that magnetars should be born rotating very fast for their fields to grow, although their braking is very efficient in the aftermath following their birth. Thus, if a magnetar is formed inside the remnant immediately after the explosion by dynamo amplification, the injection of energy (much in the same way as Ostriker and Gunn 1971 envisioned) 
is inevitable, and an initial energy loss $L_{0}=3.85 \times 10^{47}\left(\frac{B}{10^{14} G}\right)^{2}\left(\frac{1 m s}{P_{0}}\right)^{4}$ ergs $^{-1}$ and initial timescale for deceleration $\tau_{0}=0.6\left(\frac{10^{14} G}{B}\right)^{2}\left(\frac{P_{0}}{1 m s}\right)^{2} d$ can be defined for this process 4 .

Since the injected energy scales as $B^{-2}$ and the initial $\mathrm{ms}$ periods are required for field amplification to operate, the timescale for a substantial energy injection is in fact very short, of the order of $\sim$ hours (a "normal" pulsar would inject the same amount in $\sim 100-1000 y r)$. A recent work 23 showed that a bright supernova and spectra would follow, thus modified dynamical behavior at later times is naturally expected.

The injected energy will make a very young remnant to expand more quickly than the corresponding case without energy injection, making it look older in the free-expansion phase (lasting just $\sim 100 y r$ ) and later affecting the Sedov-Taylor phase, when the internal energy of the gas inside the cavity $U$ picks a term

$$
U=E-\frac{9}{32} M \dot{R}^{2}-\frac{L_{0}}{t^{-1}+\tau_{0}^{-1}}
$$

where $R$ the radius of the SNR and $M$ the mass in motion, and the last term represents the injected energy due to the magnetar formation. We refer to 4 for a thorough discussion of these features. Comparison with works dealing with standard SNR dynamics 21 15) show that the Sedov-Taylor is also modified to last longer than the case without injection, and ends after $\sim 2 \times 10^{4} E_{51}^{3 / 14} n^{-4 / 7}$ yr 4 , when the SNR enters a "snowplow" (radiative) phase. A specific search for these effects was undertaken in 22 , with negative results. However, we must remark that after $\sim 1000 \mathrm{yr}$ the speed of the ejecta is essentially the same for models with or without energy injection (Fig. 2 of Ref. [4]), making a kinematical/spectroscopical identification more difficult.

\section{The new candidate associations}

The analysis of the formerly proposed associations has been carried by several groups 4103 16, with varying results. Using the modified dynamics, we concluded in 4 that the associations AXP 1845-0258/G29,6+0,1; AXP 2259+586/CTB 109 perhaps AXP 1709-4009/G346,5-0,1 among the AXP and 1806-20/G10,0-0,3 ; SGR 1801-23 W 28 among the SGR passed this test, although they some could and have been dismissed by other reasons.

The suggestion of the newest associations CXOU J171405.7-381031/CTB 37B and XMMU J173203.3-344518/G353.6-0.7 by Halpern and Gotthelf 12 should be analyzed accordingly. Although the period and $\dot{P}$ of XMMU J173203.3-344518 could not be confirmed, Halpern and Gotthelf (2010b) have now reported a strong field value for the former, qualifying it as a magnetar. A full discussion of these SNRs 
has been addressed in several works 1020 . Fig 1 displays the Radius-Age expected from SNRs with energy injection by a magnetar for two extreme cases: a low-mass, low ISM density (labelled "low-low") case of $M=8 M_{\odot}$ and $n=0.01 \mathrm{~cm}^{-3}$ explosion; and a high-mass $M=30 M_{\odot}$ and $n=10 \mathrm{~cm}^{-3}$ one ("high-high"), hopefully bracketing the range of progenitor-ambient possibilities. The implied intervals of ages from just this plot are $800-8000 y r$ for CXOU J171405.7-381031 in CTB 37B and $1000-12000 y r$ for XMMU J173203.3-344518 in G353.6-0.7. This may seem little restrictive, but firmly excludes the estimate of 20 among others, just as a result of the modified dynamics discussed before. Since there is some information about the density in the work of 2 , we believe it is fair to consider a curve with $n=0.5 \mathrm{~cm}^{-3}$, or 50 times bigger than the "low-low" curve constituting the upper envelope. For the sake of comparison, the case of $M=10 M_{\odot}$ and $n=1 \mathrm{~cm}^{-3}$ is also plotted, if this were the case the ages wold be further tightened to $2000 \mathrm{yr}$ and $3000 y$ respectively.

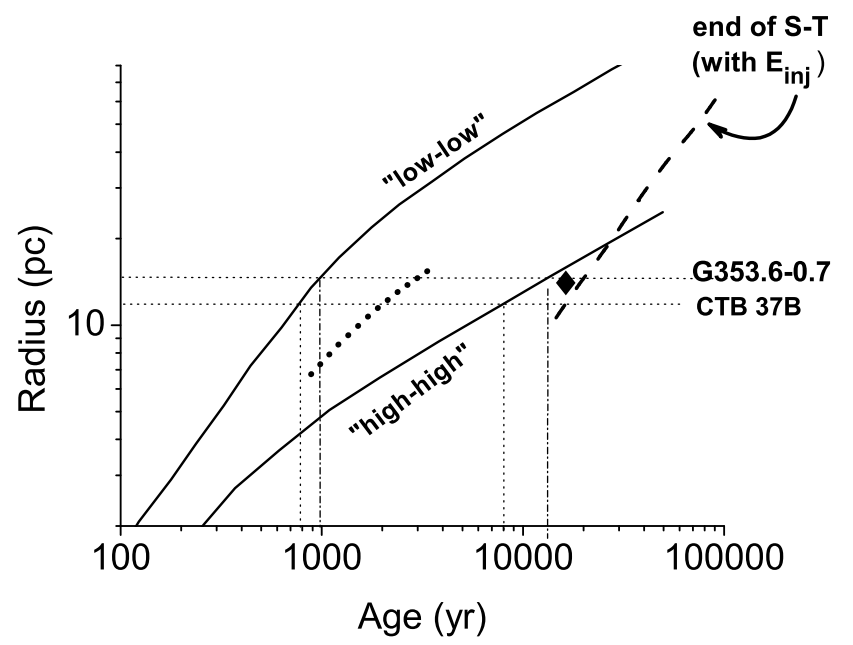

Fig. 1. The ages of magnetar-driven SNR. The limiting curves "low-low" and "high-high" are explained in the text. The dotted line corresponds to the expansion of a $M=10 M_{\odot}$ mass in a $n=1 \mathrm{~cm}^{-3}$ ambient. The end of the Sedov-Taylor phase locus is diagonal dashed line on the right of the figure. All the explosion energies were fixed to $10^{51} \mathrm{erg}$. Note that only very heavy progenitors ("high-high" or heavier) would produce the largest ages, making $C T B 37 B$ older than $10^{4} \mathrm{yr}$.

\section{Discussion and Conclusions}

The magnetar progenitors remain largely unknown, and an initial expectation of a high-mass $\left(>30-40 M_{\odot}\right) 11$ derived from an interpretation of the HI shell GSH 
288.3-0.5-28 as a wind-bubble associated to the progenitor of 1E 1048.1-5937; and later supported by the cluster analysis by 9 ?,? has been recently challenged by the identification of SGR $1900+14$ by Davies et al. (2009) with the Cl 1900+14 cluster, implying a progenitor of $<17 M_{\odot}$. Those results suggest that the difference between the events producing pulsars and magnetars should not be just related to the mass of the progenitor. We plotted in Fig. 1 the position of the shell GSH 288.3-0.5-28 interpreted as a "standard" (in the sense of the explosion energy) SNR originated from a massive $\operatorname{star}\left(\geq 30 M_{\odot}\right)$ expanding in a dense environment (diamond symbol). There is no difficulty with the energetics, and the inferred age is $\sim 15000 \mathrm{yr}$, provided the modified dynamics is employed.

We have inferred ages for G353.6-0.7 and CTB 37B which are much smaller than the values derived within conventional models. Refining the broad interval to include a ISM density closer to usual values (as directly measured for CTB 37B by 2 ), the figures for the ages are still low and would not change much unless the mass of the progenitor was $\geq 4 \times 10 M_{\odot}$ (see Fig. 1). The age of this remnant is closer to the "old" estimation of $\sim 1500 y r 7$, but for quite different dynamical reasons. This age allowed a prediction of the $\dot{P}$ value, provided the characteristic age is close to the actual value, of $\dot{P} \sim 4 \times 10^{-11} s^{-1}$, which in turn predicts a magnetic field strength of $B=4 \times 10^{14} G$, almost exactly the values obtained by Halpern and Gotthelf 13 for CXOU J171405.7-381031 right after the Cesme event (!). It is also interesting to note that younger objects easy the requirements for energizing the $\mathrm{TeV}$ scale, as observed by the HESS Collaboration 2. electrons still "live" without being cooled (12) or even the SNRs themselves contribute, because they are actually younger than they seem.

To summarize, we believe that we are not dealing with ordinary remnants, but rather with a very special variety of them, the magnetar-driven ones, a natural result of dynamo field growth to the $10^{14}-10^{15} G$ scale. It is in this framework that associations need to be analyzed. An alternative picture in which field values result from flux conservation would not render substantial dipole energy injection.

\section{Acknowledgments}

We acknowledge the financial support received from the Fundação de Amparo à Pesquisa do Estado de São Paulo and CNPq Agency (Brazil). JEH wishes to thank the hospitality of the Organizers of ASTRONS2010.

\section{References}

\section{References}

1. F. Aharonian et al. (H.E.S.S. Collaboration), $A \mathscr{E} A$ 486, 829-836 (2008a).

2. F. Aharonian, et al. (H.E.S.S. Collaboration), A\&A 477, 353-363 (2008b).

3. A. Ankay, O.H. Guseinov, M.A. Alpar and S.O. Tagieva, arXiv:astro-ph/0110092 (2001).

4. M.P. Allen and J.E. Horvath, Astrophys. J. 616, 346-356 (2004) 
5. J.L. Bibby et al.MNRAS 386, L23-L27 (2008).

6. N.V. Ardeljan, G.S. Bisnovatyi-Kogan and S.G. Moiseenko, MNRAS 359, 333-344 (2005).

7. D.H. Clark and F.R. Stephenson, The Observatory 95, 190-200 (1975)

8. D. Davies, D.F. Figer, R-P. Kudritzki, C. Trombley, C. Kouveliotou and S. Wachter,Astrophys. J. 707, 844-851 (2009)

9. D.F. Figer, F. Najarro, T.R. Geballe, R.D. Blum and R-P. Kudritzki,Astrophys. J. 622, L49-L52 (2005).

10. B.M. Gaensler, P.O. Slane, E.V. Gotthelf and G. Vasisht, Astrophys. J. 559, 963-972 (2001).

11. B.M. Gaensler et al. Astrophys. J. 620,L95-L99 (2005).

12. J.P. Halpern and E.V. Gotthelf,Astrophys. J. 710, 941-947 (2010a).

13. J.P. Halpern and E.V. Gotthelf, arXiv:1008.2558 (2010b).

14. M. Hamuy, Astrophys. J. 582, 905-914 (2003)

15. D. M. G. C. Luz and D.L. Berry, MNRAS 306, 191-198 (2010).

16. D. Marsden, R.E. Lingenfelter, R. E. Rothschild and J.C. Higdon, Astrophys. J. 550, 397-309 (2001).

17. M.P. Muno et al. Astrophys. J. 636, L41-L44 (2006).

18. J.P. Ostriker and J.E. Gunn, Astrophys. J. 164, L95-L104 (1971).

19. T. A. Thompson, P. Chang and E. Quataert, Astrophys. J. 611, 380-393(2004).

20. W.W. Tian et al., Astrophys. J. 712, 790-796 (2010).

21. J.K. Truelove and C.F. McKee, Astrophys. J.Supp. 120, 299-326 (1999).

22. J. Vink and L. Kuiper, MNRAS 370, L14-L18 (2006).

23. S. Woosley,Astrophys. J. 719, L204-L207 (2010). 